\title{
INVESTIGATING THE EFFECT OF A RAISED CYCLE TRACK, PHYSICAL SEPARATION, LAND USE AND NUMBER OF PEDESTRIAN ON CYCLISTS' GAZE BEHAVIOR
}

\author{
Gukhwa JANG*, Saehoon KIM \\ Department of Landscape Architecture, Urban Design Concentration, Graduate School of Environmental Studies, \\ Seoul National University, Seoul, Korea
}

Received 11 July 2018; accepted 14 March 2019

\begin{abstract}
Contemporary cities are home to an increasing number of cyclists. The gaze behavior of cyclists has an important impact upon cyclist safety and experience. Yet this behavior has not been studied to access its potential implications for urban design. This study aims to identify the eye-gaze pattern of cyclists and to examine its potential relationships with urban environmental characteristics, such as a raised cycle track, physical separation, land use, and number of pedestrian. This study measured and analyzed 40 cyclist's gaze patterns using an eye tracker; the results were as follows. First, cyclists presented a T-shaped gaze pattern with two spots of frequent eye fixation points; the pattern suggests that it may benefit cyclists with greater safety and better readiness of road situation to avoid crashes. Second, more active horizontal gaze dispersion within the T-shaped gaze pattern was observed when participants cycled on a shared and non-raised bikeway. This indicates that there is a more suitable gaze behavior with different gaze limitations depending on the environmental characteristics. Therefore, bicycle facilities need to be constructed according to the consideration of the T-shaped gaze area and the change in cyclists' gaze behavior in each environment to increase the effectiveness of bicycle facilities.
\end{abstract}

Keywords: bicycle safety, cycle-friendly design, urban bikeway, scan strategy, gaze area, gaze dispersion, eye tracker.

\section{Introduction}

Contemporary cities are home to an increasing number of cyclists and non-motorized vehicles. Cycling is an important form of transportation in many places around the world and cycling makes up a small but significant amount of total time and distance traveled for commuting, exercising, and recreational purposes. Many cities are investing in the provision of cycling infrastructure and bike-related services to encourage cycling. Many experts in the field of transportation planning and urban design have proposed urban design guidelines to implement effective bicycle infrastructure and amenities towards the goal of creating a bike-friendly city including a design standard for bicycle lanes and signs (such as placement, shape, and color).

However, cyclists are still frequently exposed to risky situations in dense urban environments. According to the NHTSA's Traffic Safety Facts (National Highway Traffic Safety Administration), the number of fatalities from bicycle accidents in the U.S was 818 in 2015. The rate has increased at about $2 \%$ each year over the past ten years.
The current bike infrastructure may not be sufficient to prevent these hazards in cycling without considering the causes of accidents. According to previous studies, the lack of adequate visual attention to the road condition and nearby vehicles has been known as a major factor that causes traffic accidents (Ballham, Absoud, Kotecha, \& Bodiwala, 1985; Liu, Shen, \& Huang, 1995; Vanparijs, Panis, Meeusen, \& de Geus, 2016). The built environment characteristics of cities are a major factor that affects cyclists' visual behavior (Crundall, Van Loon, \& Underwood, 2006; Bendak \& Al-Saleh, 2010; Edquist, Horberry, Hosking, \& Johnston, 2011). Since bikers make perceptual decisions in cities with heterogeneous environments, the built environments act as both positive stimuli and as a disorganized distractor. As cyclists are distracted from information unrelated to cycling, there may be a growing possibility that necessary information for cycling is missed. Consequently, they might make bad decisions and the probability of accidents could increase (Hajime, Atsumi, Hiroshi, \& Akamatsu, 2001). Thus, an environmental design that leads to adequate visual behavior is required

${ }^{*}$ Corresponding author. E-mail: jangguka@naver.com 
for bicycle safety and an examination of which environmental factors attract cyclists' attention is necessary.

Recently, previous studies have focused on the interactions between the environment and the gaze movement by analyzing gaze allocation to specific environmental factors (Vansteenkiste, Cardon, D’Hondt, Philippaerts, \& Lenoir, 2013; Vansteenkiste, Zeuwts, Cardon, Philippaerts, \& Lenoir, 2014; Vansteenkiste, Cardon, \& Lenoir, 2015; Vansteenkiste, Zeuwts, Cardon, \& Lenoir, 2016; Ahlstrom, Kircher, Thorslund, \& Adell, 2016; Mantuano, Bernardi, \& Rupi, 2017; Zeuwts et al., 2016). Cyclists generally do not recognize all information at the same time due to their limited recognition capability, so only specific information that is important for driving is selected and recognized (Horrey, Wickens, \& Consalus, 2006). Several studies have suggested that particular environmental areas that will likely have more required information play a significant role for cyclists (Vansteenkiste et al., 2013, 2016; Ahlstrom et al., 2016; Mantuano et al., 2017). Cyclists' fixations were dominantly located at the road and the Focus of Expansion (FOE) that represents the point of cycling direction. Furthermore, previous studies have implied that the greater mental load was found at specific road conditions, often leading to gaze movement from external areas to functional areas such as the road or Focus of Expansion (FoE) (Chapman, Underwood, \& Roberts, 2002; Bendak \& Al-Saleh, 2010; Hosking, Liu, \& Bayly, 2010; Vansteenkiste et al., 2013, 2014; Frings, Parkin, \& Ridley, 2014; Mantuano et al., 2017). The gaze shift was found according to some environmental factors such as road quality and width (Vansteenkiste et al., 2013, 2014). These papers could provide objective results on cyclists' gaze behavior by using an eye-tracker. The direct measurement of eye movement was useful to better investigate how cyclists look around the urban environment.

However, there are limitations to understand cyclists' gaze behavior and to apply previous findings to the bicycle facility design due to the condition of laboratory tests and the insufficient exploration of other environmental factors. Cycling in controlled and simulated conditions might not be suitable to represent natural gaze movement (Vansteenkiste et al., 2013). The task difficulty between a simulated experiment and a field test may be different. The participants in a simulated experiment may not feel as nervous as those in a field experiment although a similar risky situation has arisen. Then, this different task difficulty can interrupt the reflection of the effect of an environmental factor on gaze behavior. Another limitation is the lack of considering other environmental effects on gaze behavior such as the presence of exclusive bike lanes, a raised cycle track, number of pedestrian, and land use. Although the effects of factors such as the width and quality of bicycle lanes were discovered, it is not enough to represent complex road environments.

Therefore, with the aim of proposing an efficient standard for bike infrastructure design, we investigated the gaze pattern of cyclists in urban areas. Cyclists' gaze patterns in urban situations were empirically investigated by analyzing gaze allocation and gaze dispersion using an eye tracker. By examining their gaze patterns, the frequently perceived range and spots in urban areas were found when cycling. Moreover, the relationships between urban environmental characteristics and bikers' gaze strategies were investigated to evaluate the potential effects of urban characteristics on gaze behavior. The following environmental characteristics were associated with gaze pattern in this paper: (1) the presence of a raised cycle track, (2) physical separation (the presence of exclusive bike lanes), (3) land use, and (4) number of pedestrian. We expected that cyclists had a specific gaze behavior unlike driver's one and the gaze shift would be found according to the difference of urban characteristics.

\section{Literature review}

Road conditions such as road width, surface quality, and the presence of exclusive bike lanes have been known to be primary factors influencing the gaze pattern of cyclists. For instance, Vansteenkiste et al. $(2013,2014)$ suggested that when cycling on low-quality roads, bikers are visually more focused on the road area compared to the external area (including street objects, trees, and vehicles and pedestrians) since a danger of falling off the bicycle could be greater due to the uneven road surface or the narrow road width. On the other hand, the eye movement of participants became more active at a higher quality of bike lanes due to a slight need to attend to the road condition (Vansteenkiste et al., 2014). Moreover, Mantuano et al. (2017) indicated that the absence of a physical separation in bike lanes resulted in a lack of detection of unexpected hazards. As the attention of bikers increases to avoid collisions with nearby road users, the time to perceive a danger might comparatively decrease. In contrast, in exclusive bike lanes, cyclists could spend more time detecting the hazards due to the growing spare time with a lower collision risk.

Visual clutter is another expected environmental factor related to the visual behavior of bikers. Disorderly, sometimes unnecessary or uncontrolled clutter in urban areas captures the attention of bikers and occupies their perceptual space, leading to delayed time for behavior judgment and greater visual stress during biking. Some examples of visual clutter are different land uses, the presence of parking lots, and roadside advertisements. The visual complexity of the surrounding environment provides much visual information that cyclists need to judge or remember, which may lead to missing important visual information, such as pedestrians and the condition of the road surface.

In addition, bikers can easily be distracted by a nonrelevant distractor when their perception is overly occupied by visual clutter. According to Perez and Bertola (2011), drivers gazed at the roadside more frequently and the number of fixations on external areas decreased in the condition of high visual clutter. Accordingly, the authors 
suggested that the high visual clutter increased the mental load, and then drivers paid more attention to the road situation. On the other hand, Hughes and Cole (1986) assessed the difference in visual behavior in residential vs. commercial areas. The results of this study indicated that drivers had fewer fixations on driving-related information and more fixations on vehicles and pedestrians and adverts in commercial areas. Besides, visual clutter affected not only gaze allocation, but also gaze dispersion. The more complex the road environment, the wider and more active the drivers' horizontal gaze dispersion since too much unnecessary information attracted cyclists' attention (Chapman et al., 2002; Hosking et al., 2010).

According to previous studies, road conditions and visual clutter is primary factors to influence on cyclists' gaze pattern. However, many environmental characteristics' effects on gaze behavior have not been discussed. For example, a raised cycle track and an exclusive bike lane are also important road characteristics to determine road conditions in addition to the road width and the road surface quality. Moreover, land use and the number of pedestrian are major determinants to change visual clutter of road situations. Thus, we focused on the potential relationship between gaze behavior and environmental factors for which the effect has been unknown and expected to contribute to cyclists' visual characteristics significantly; a raised cycle track and physical separation as factors of bicycle road condition and land use, as well as number of pedestrian as one of the parameters of visual clutter.

\section{Research methods}

\subsection{Cyclists selection and the study area}

\section{Participants}

A total of 50 participants (25 males and 25 females; $22-26$ years of age) took part in this experiment. We recruited participants from the website of Seoul National Univer- sity. Most participants have cycled once a month $(n=20)$ or several times a year $(n=15)$ for exercise and recreation. Only individuals who did not wear glasses were selected for accurate measurements and it was excluded from experiment that a participant was not calibrated or did not fit the experimental equipment.

\section{Apparatus}

Gaze movements were measured using a head mount eye tracker (Tobii Pro Glasses2) with a $50 \mathrm{~Hz}$ sampling rate. By using this apparatus, we could measure 50 eye positions per second. The device consists of four eye cameras and one scene camera. Eye cameras used near-infrared illumination to make reflection on the eyes, then tracking the eye locations. The road situation was recorded with a camera attached to eye tracker and the recorded scene had a visual angle of $52^{\circ}$ vertically and $82^{\circ}$ horizontally.

\section{Cycling route}

A cycling route was chosen in Songpa-Gu, Seoul, and Republic of Korea. The distance of the whole cycling route was $6 \mathrm{~km}$ long, including training and actual routes. Training routes were added at the beginning of an experiment and between actual routes to adapt participants to road situations for determining cyclists' natural gaze behavior (see Figure 1). When cyclists rode a bike on the training routes, cyclists' gaze behavior was not analyzed. We used only the eye-movement data measured on the actual routes. The bicycle road on the whole route was one-way, colored and with signage. Also, no parking was allowed along the route, and the cycling direction was the same as the driving direction.

We divided the actual routes into six sections according to the urban environments (see Table 1). Section 1, 2 and 3 had a non-raised cycle track, while section 4, 5 and 6 were located on the road with a raised cycle track. Section 2 and 3 were physically separated from motor traffic by bollards, whereas the other sections were only visually

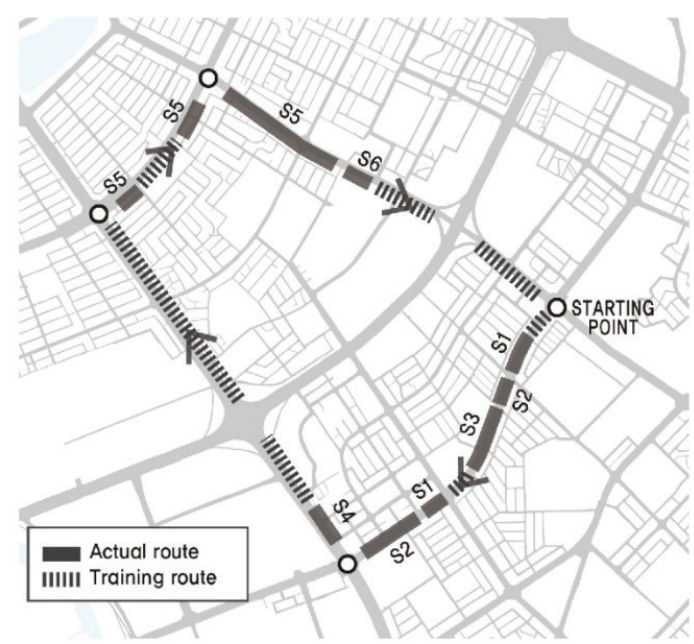

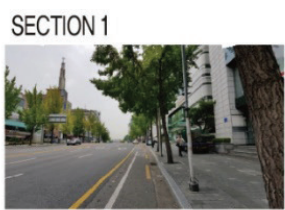
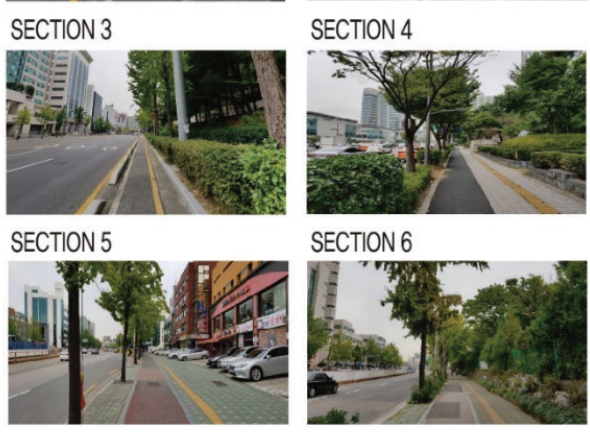

Figure 1. Cycling Route. Section 1 (upper left), Section 2 (upper right), Section 3 (middle left), Section 4 (middle right), Section 5 (bottom left) and Section 6 (bottom right) (source: author, 2017) 
Table 1. The characteristics of cycling route per each section

\begin{tabular}{|c|c|c|c|c|c|}
\hline & Distance & $\begin{array}{c}\text { Presence of } \\
\text { raised cycle track }\end{array}$ & Physical Separation & $\begin{array}{c}\text { Land use } \\
\text { pedestrian }\end{array}$ \\
\hline Section 1 & $289 \mathrm{~m}$ & A non-raised cycle track & Shared bike lanes & Built-up area & Low \\
\hline Section 2 & $378 \mathrm{~m}$ & A non-raised cycle track & Exclusive bike lanes & Built-up area & Low \\
\hline Section 3 & $231 \mathrm{~m}$ & A non-raised cycle track & Exclusive bike lanes & Greenery area & Low \\
\hline Section 4 & $116 \mathrm{~m}$ & A raised cycle track & Shared bike lanes & Greenery area & High \\
\hline Section 5 & $917 \mathrm{~m}$ & A raised cycle track & Shared bike lanes & Built-up area & Low \\
\hline Section 6 & $73 \mathrm{~m}$ & A raised cycle track & Shared bike lanes & Greenery area & Low \\
\hline
\end{tabular}

separated by a colored-lane from vehicles and pedestrians. In terms of land use, sections 1, 2 and 5 were placed in built-up areas and section 3, 4 and 6 were located in green areas. Lastly, the number of pedestrian of all sections was low, apart from section 4.

\section{Procedure}

The experiment was conducted from August 3 to 12, 2017, and September 16 to 24,2017 . We did not perform the experiment on rainy days and at rush hour times for safety reasons. In addition, overly sunny days were not suitable for the experiment because sunlight interferes with eye measurements through near-infrared illumination.

First of all, participants completed a questionnaire on age and frequency of cycling and were given general information about experimental procedures. Following this, participants wore the eye tracker, a helmet and a visor for safe and accurate measurements. A visor was used to cover the sunlight. After the eye tracker was calibrated, the participants were asked to cycle in accordance with the experimental route and with no distracting behavior, such as listening to music or using a cell phone. We also asked participants for cycling at a speed of about $3 \mathrm{~m} / \mathrm{s}$. All participants rode the same bicycle for an equal cycling condition and the experiment lasted for approximately 50 minutes.

\subsection{Measurement of environment characteristics}

Environmental characteristics were categorized according to four factors: the presence of a raised cycle track (a raised cycle track vs. a non-raised cycle track), physical separation (exclusive bike lanes vs. shared bike lanes), land use (greenery area vs. built-up area), and number of pedestrian (high vs. low).
First, a raised cycle track was determined by the height of the bicycle lane compared to that of the motorway (see Figure 2). Raised cycle tracks were located at the same level of the sidewalk, with a greater height of roughly $0.15 \mathrm{~m}$ from the motorway. This vertical separation prevented car access. On the other hand, a non-raised cycle track referred to a placement with the same height as the motorway. The cycle track was separated from drivers by only a road marking or bollards and from pedestrians by a raised curb. There was no difference in the arrangement of road types (including the motorway, bike lane, and sidewalk) between two road level types.

Second, the physical separation was divided according to the presence of the physical separation with vehicles and pedestrians. In the present study, the exclusive bike lanes restricted the access of drivers by bollards or the curb. By contrast, the shared bike lanes had only road markings to distinguish each road user's areas, leaving the possibility of collision.

Third, land use was categorized as built-up and greenery areas to investigate the effect of visual complexity differences. Located on the commercial street, the built-up area included diverse distractors, such as billboards, shop windows, and street events. By contrast, the greenery area in the urban environment had a relatively monotonous streetscape, leading to lower visual clutter than that of other urban streets.

Finally, number of pedestrian was measured in two degrees (high, low). While the high number of pedestrian amounted to roughly 1200 individuals per hour at non-peak time, the low number of pedestrian was approximately half that amount. The two conditions were measured on the raised and shared bike lanes.
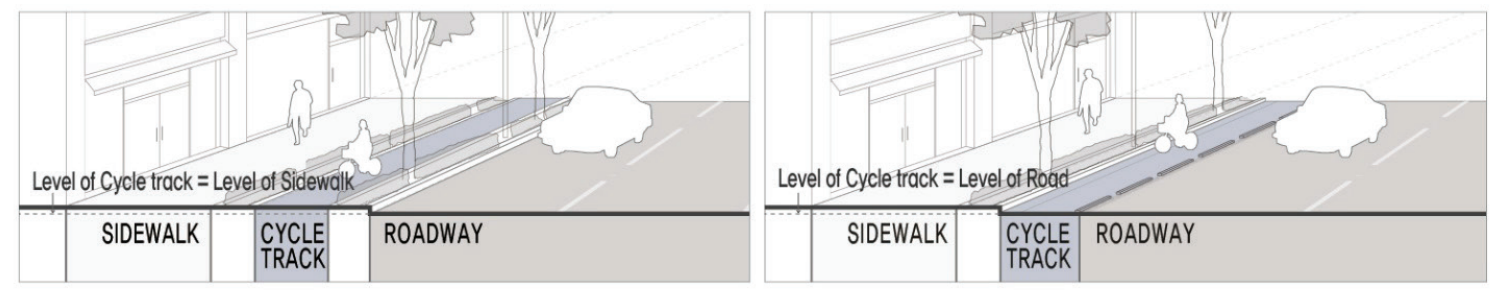

Figure 2. A raised cycle track (left) and A non-raised cycle track (right) 


\subsection{Analytical methods}

The data of 40 participants was analyzed, excluding the data of 10 individuals which had an ETR (Eye Tracking Ratio) below $80 \%$. With asking for a speed limit, the cycling speed between participants was not significantly different.

From the raw data, we extracted a fixation data using the Tobii pro glasses analyzer program, in order to encode the gaze data with a method of fixation by fixation which use only fixation data as valid one. Before the extraction, method of data interpolation was used to fill the gap of two recorded gaze within $75 \mathrm{~ms}$, due to missing data from sunshine and blinks. After then, the gaze data was categorized as fixation or saccade using velocity-based algorithms, which classify a type of gaze by the velocity of the directional shifts of the eye. Subsequently, this extracted data was manually encoded to each road factor (FoE, all road types, building, tree, street furniture, vehicles and pedestrians). A gaze was not encoded when unexpected road events occurred (e.g. a sudden approach of vehicles and pedestrians, at intersection and crosswalks). Lastly, this encoded data was divided into six sections and analyzed following two methods.

\section{Gaze dispersion and fixation location (\%) per road factor}

In this paper, two analysis methods were used. First, a method of grid analysis was used to examine the spread of gaze location and the frequent gaze spot or area. It can be described as follows. The criterion of cyclists' visual scene was set up as horizontally $210^{\circ}$ and vertically $120^{\circ}$, in order to include not only the basic range of eye movement (about horizontally $200^{\circ}$ and vertically $110^{\circ}$ ) but some additional range of the head movements (horizontally and vertically $10^{\circ}$ ). Then, the visual scene was divided by a $20 \times 10$ grid per $10^{\circ}$ and an average fixation duration percentage (\%) per grid was measured. The central point of grid (zero point) was defined to be FoE. This is because the vertical position of the FoE is equal to the eye level and the horizontal position of the FoE is in the middle of the visual scene. The result from this method can be used to determine the horizontal gaze dispersion, i.e. by how much the gaze is to the active right (road-side) or to the left (sidewalk-side). On the other hand, vertical gaze dispersion refers to the distance of the target region of the gaze (Hosking et al., 2010).

Second, we divided the road environment into six road factors to measure which factor in road situations cyclists primarily focus on: FoE, all road type, building, tree, street furniture, and other road users (vehicles and pedestrians) (see Figure 3). FoE (Focus of Expansion) means a point that cyclists' gaze is focused on for steering and guidance. All road types include roadway, sidewalk and bicycle lanes. Street lamp, signs, a telephone pole, fence and bus stop were classified as street furniture. In addition, both stopped and moving road users (vehicles and pedestrians) were measured.

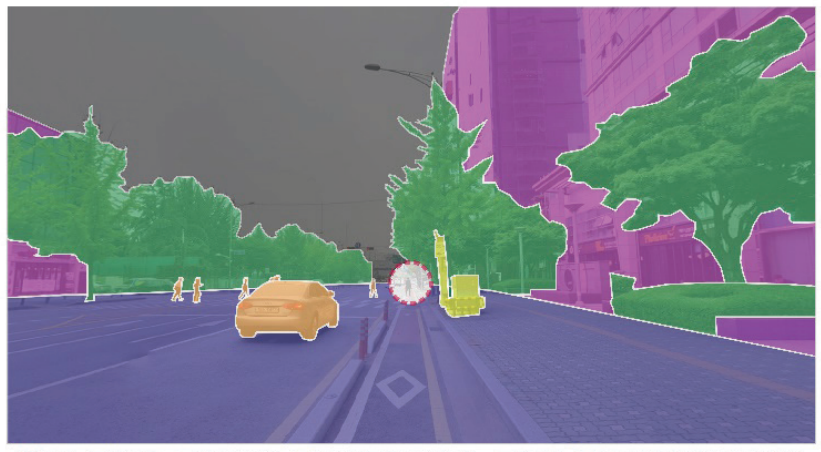

FoE $\square$ ROAD $\square$ BUILDING $\square$ STREET FURNITURE $\square$ TREE $\square$ VEHICLES PEDESTRIANS

Figure 3. Classification of road factors

\section{Statistics}

The difference in gaze allocation and dispersion for each urban characteristic was examined by comparing each section: (1) the presence of a raised cycle track: section 1 (a non-raised cycle track) vs. section 5 (a raised cycle track); (2) physical separation: section 1 (shared bike lanes) vs. section 2 (exclusive bike lanes); (3) land use: section 2 (built-up area) vs. section 3 (greenery area); and (4) number of pedestrian: section 4 (high) vs. section 6 (low). These were comprehensively tested using the paired t-test in SPSS 22. The significance level was set to $\mathrm{p}<0.05$.

\section{Results}

\subsection{Gaze pattern: T-shaped gaze area with two gaze spots}

\section{Spread of gaze location}

The gaze dispersion was analyzed by horizontal and vertical fixation locations (see Figure 4). Most fixations were horizontally concentrated within the narrow visual field to range between $-10^{\circ}$ and $10^{\circ}(36.80 \%)$, including FoE and bicycle road area. Even if most fixations were evenly distributed in both directions, the greater number of fixations was located on the left side (35.05\%) than the right side (25.17\%). This suggested that cyclists paid more attention to the road-side than the sidewalk-side. In addition, there seemed to be specific spots of eye movement $\left( \pm 30^{\circ}\right)$ at which participants' gaze was frequently fixated to keep aware of the current road situation rather than to navigate or steer. On the other hand, most vertical fixations were located below eye-level (zero degrees) and peaked at the range from $-10^{\circ}$ to $0^{\circ}(25.31 \%)$. The fixations near the region of bike lanes were as frequent as those on the distant region.

Moreover, there were two spots of frequent eye fixations, accounting for over half of the whole fixation duration (61.42\%): FoE ( $0^{\circ}$ vertically and horizontally) and a particular spot on the bicycle road (the range from $-20^{\circ}$ to $-30^{\circ}$ vertically and $0^{\circ}$ horizontally). 
Overall, participants' gaze distribution with active eye movement between distant and nearby regions displayed a T-shaped gaze area, having an occasional horizontal movement of fixations at eye level (see Figure 5). Regardless of the road characteristics, we could identify the Tshaped gaze area pattern in all cycling sections, representing a slight variation in the gaze area.

\section{Percentage of fixation duration for each road factor}

In Table 2, the percentage of fixation duration for each road factor is presented. \% all road types $(46.7 \pm 23.0)$ accounted for approximately half of the whole duration, and $\%$ FoE was the second largest $(26.9 \pm 17.9)$. Fixations on the FoE and road areas were dominant in proportion, with at least $73.6 \%$ of the total fixation duration. On the other hand, \% buildings (9.6 $\pm 8.7 \%)$, \%vehicles and pedestrians $(8.3 \pm 13.8), \%$ trees $(6.8 \pm 8.1)$, and \% street objects $(2.2 \pm 3.0)$ were observed, revealing the total percentage of $27 \%$ in the external areas.

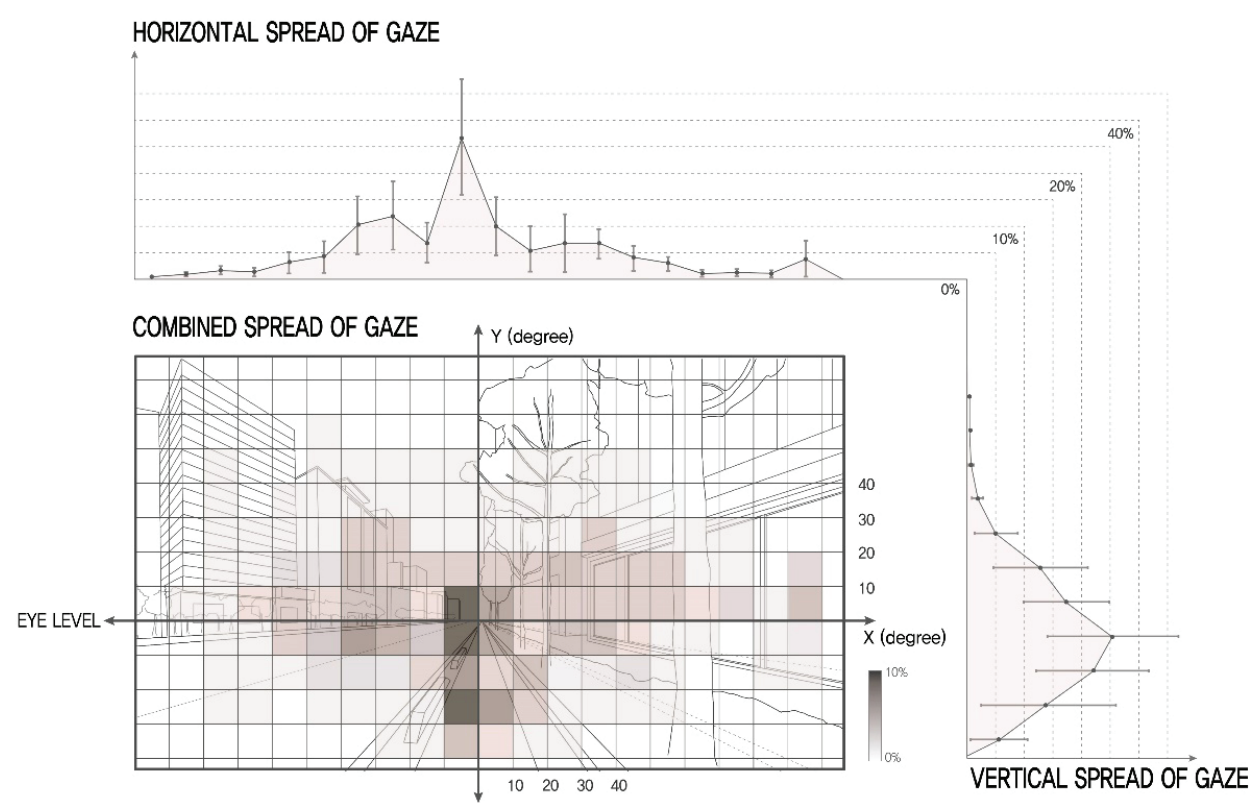

Figure 4. Horizontal, vertical and combined gaze dispersion
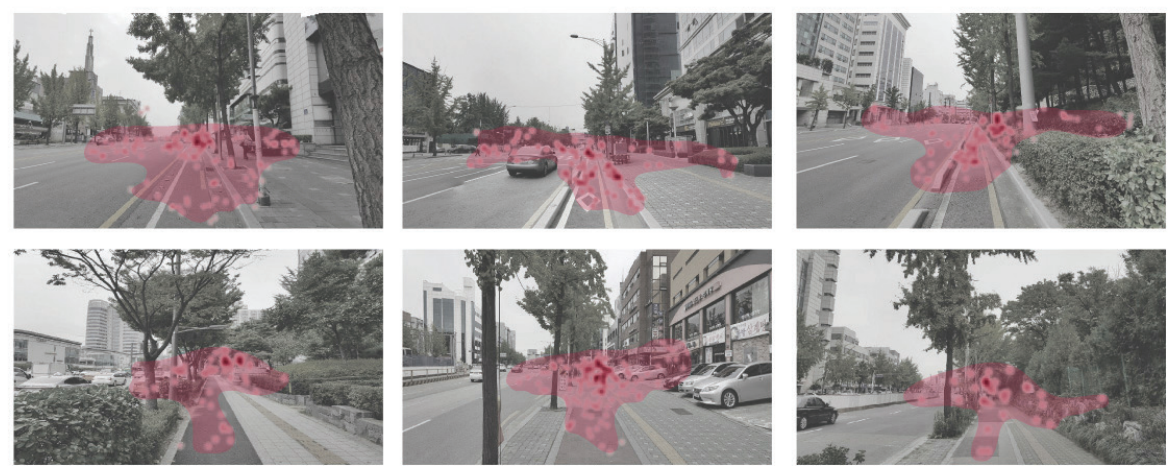

Figure 5. The representation of an T-shaped gaze area pattern on each section. Section 1 (upper left), Section 2 (upper middle), Section 3 (upper right), Section 4 (bottom left), Section 5 (bottom middle) and Section 6 (bottom right)

Table 2. Mean values and standard deviations of fixation duration percentage (\%) for each road factor

\begin{tabular}{|c|c|c|c|c|c|c|}
\hline & \multicolumn{6}{|c|}{ Road Factors } \\
\cline { 2 - 7 } & FoE & All road types & Building & Tree & Street Furniture & Vehicles and pedestrians \\
\hline Mean (\%) & 26.9 & 46.7 & 9.6 & 6.8 & 2.2 & 8.3 \\
\hline SD & 17.9 & 23.0 & 8.7 & 8.1 & 3.0 & 13.8 \\
\hline
\end{tabular}




\subsection{Effects on gaze pattern from urban characteristics: a raised cycle track, physical separation, land use and number of pedestrian}

\section{Spread of gaze location}

In this section, the horizontal and vertical dispersion for each urban characteristic was analyzed (see Figure 6 \& 7). Although the vertical gaze distribution seemed to show a slight difference, there was no significant difference according to the urban environmental characteristics ( $\mathrm{p}<$ $0.05)$. On the other hand, the effect on the horizontal gaze dispersion was found with the difference in the presence of exclusive bike lanes and s raised bikeway. When participants cycled on a raised cycle track, fixations were more concentrated on the FoE $(t=-2.39, p=0.02)$. Similarly, a higher percentage of gaze on the FoE was found in exclusive bike lanes $(t=-2.45, p=0.02)$, although they ex- hibited no difference in gaze allocation for all road factors (see Figure 8).

\section{Percentage of fixation duration for each road factor}

In Figure 8, the percentage of fixation duration for each road factor is presented. Overall, the percentage of FoE was not affected by differences in environmental conditions, maintaining a constant percentage (one third of all fixations). \% road was changed with only a difference in the bicycle road level.

When participants cycled at a non-raised cycle track, \% all road types increased by nearly ten percent $(\mathrm{t}=4.12, \mathrm{p}=$ $0.00)$ and a growing percentage in buildings $(t=-7.19$; $\mathrm{p}=0.00)$ and vehicles and pedestrians $(\mathrm{t}=-3.50, \mathrm{p}=0.00)$ was found. In contrast, looking at street objects decreased by three percent $(t=5.38, p=0.00)$ and no significant difference was found for $\%$ FoE and $\%$ trees $(p<0.05)$.

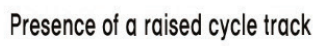

Physical separation

Land Use

Number of pedestrian
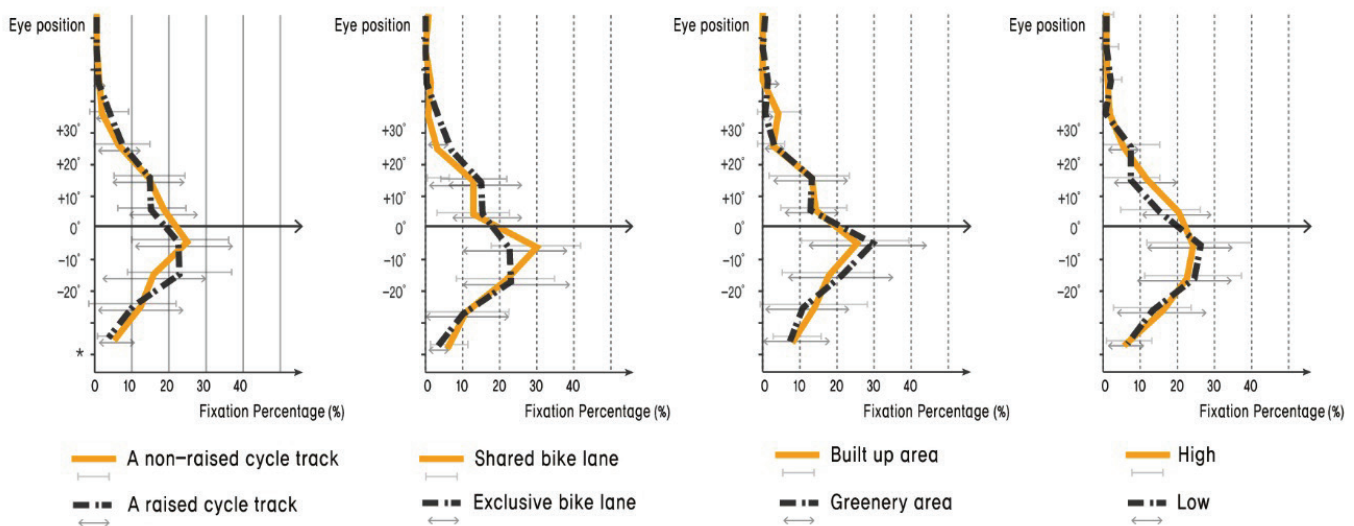

Figure 6. Vertical spread of fixation duration (\%) for the presence of a raised cycle track, physical separation, land use, number of pedestrian. And ${ }^{*}$ presents to have significant difference
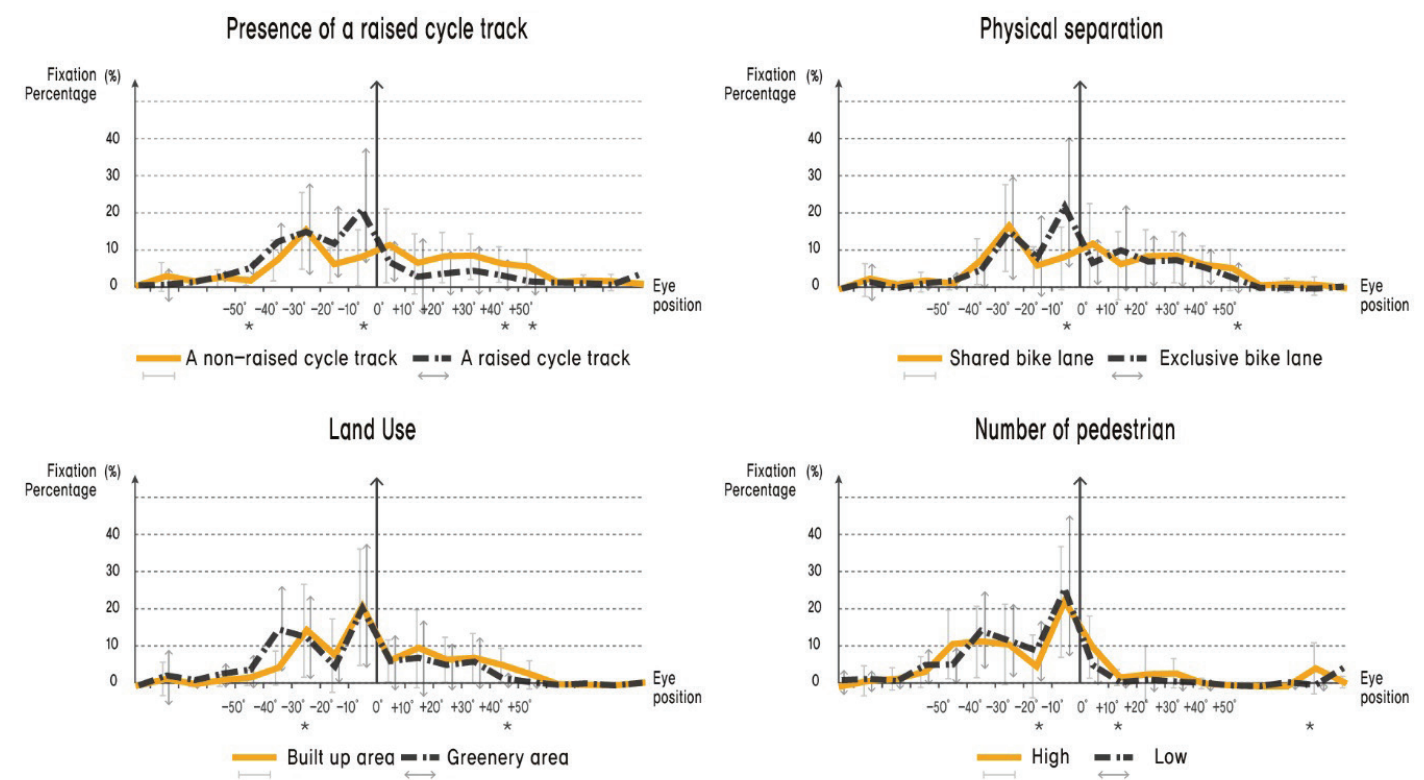

Figure 7. Horizontal spread of fixation duration (\%) for the presence of a raised cycle track, physical separation, land use, number of pedestrian. And ${ }^{*}$ presents to have significant difference 
Presence of a raised cycle track

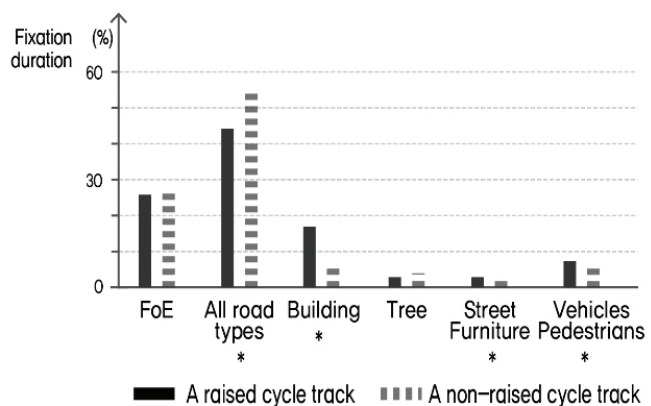

Land use

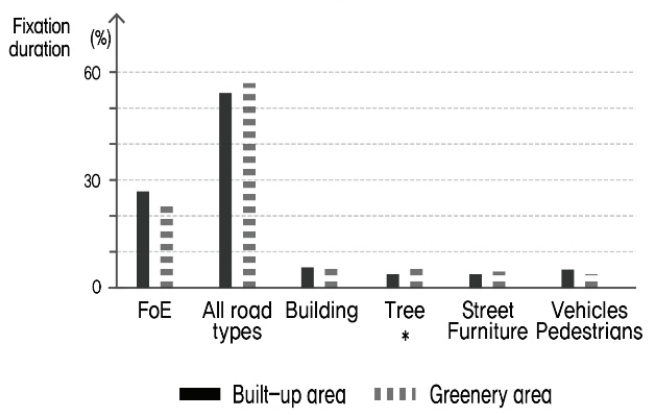

Physical seperation

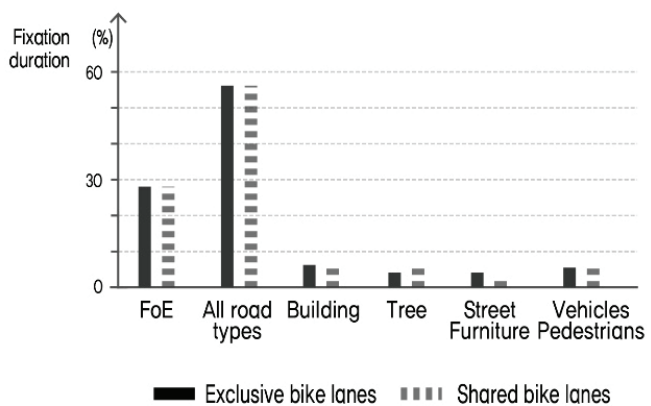

Number of pedestrian

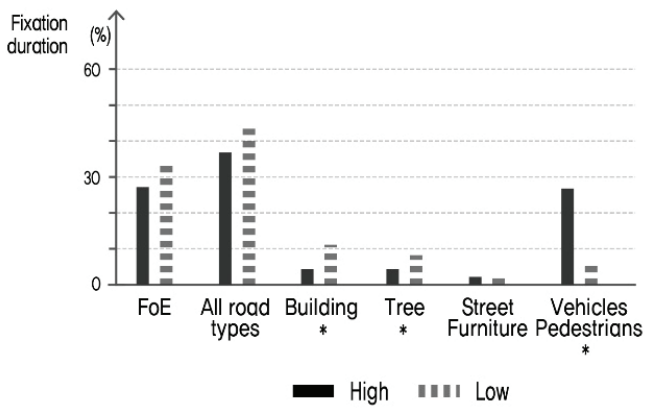

※ Fixation duration $(\%)$ = percentage of fixation duration in each road factor of the total fixation duration

Figure 8. Mean fixation duration percentage (\%) in FoE, all road types, buildings, trees, street objects, vehicles, \& pedestrians. And ${ }^{\star}$ presents to have significant difference

While percentage of gazing toward vehicles \& pedestrians increased considerably by 25 percent $(t=7.78$, $\mathrm{p}=0.00)$ on road with a higher number of pedestrian, fixations on $\%$ buildings $(\mathrm{t}=-6.47, \mathrm{p}=0.00)$ and $\%$ trees $(\mathrm{t}=-5.23, \mathrm{p}=0.00)$ decreased. The absence of exclusive bike lanes $(\mathrm{t}=-2.02, \mathrm{p}=0.05)$ and the difference in land use $(\mathrm{t}=-2.71, \mathrm{p}=0.01)$ showed no significant effects on each $\%$ road factor, except for $\%$ trees.

\section{Discussion}

\section{Cyclists' T-shaped gaze pattern}

From the presented results, we concluded that cyclists had a T-shaped gaze area with two frequent eye fixation points for steering. The cyclists' gaze pattern of cyclists had three characteristics.

First, a T-shaped gaze area of bicyclists was found, suggesting the road factors within this area tended to be more easily accepted. Cyclists had horizontally concentrated and vertically wider movement below eye-level. In other words, cyclists tended to allocate their attention to a nearer region (the road) as much as a distant region (FoE) with less time spent looking from side to side than in drivers' eye movement. The pattern had a considerable difference compared with that of drivers, which was a horizontally broad and vertically narrow eye movement.

This tendency might be caused by the task difference related to road gaze and the restriction of the visual field. It may be advantageous for drivers to have a wider road scan strategy for keeping in lanes or changing and maintaining a lateral distance from other drivers. In contrast, bicycle paths only have one lane (or at most two) in urban areas, so cyclists do not need to change their lanes or maintain a lateral distance from other cyclists. Consequently, active horizontal eye movement is not necessarily required in cyclists. In addition, cyclists have an unrestricted view, while drivers' view is limited by their dashboard (Vansteenkiste et al., 2014). Drivers' restricted road scene leads them to check only the distant region. However, cyclists can actively shift their gaze between distant and nearby regions. Accordingly, a distinct gaze pattern of cyclists with an occasional gaze shift to external areas mostly at eye level facilitated fixations in a T-shaped gaze area.

Second, cyclists had two gaze spots of frequent eye fixations in accordance with finding of previous studies (Vansteenkiste et al., 2013, 2014), while drivers' fixations were particularly concentrated on a spot (FoE) with occasional gaze toward the road or external areas (Mourant \& Rockwell, 1970; Helander \& Söderberg, 1972). This difference can be explained via the two-level model of driver steering behavior (Donges, 1978). The model argues that there are two gaze levels for steering: guidance and stability. Regarding visual attention, drivers gaze at distant regions for guidance and nearby regions for stability. The gaze amount for each region is adjusted in accordance with the road situation. When cyclists have a high demand for guidance, their gaze toward a distant region, such as FoE, may increase. When the demand for stability exceeds 
that for guidance, the gaze shifts from distant to nearby regions (Pelz \& Rothkopf, 2007; Vansteenkiste et al., 2013).

According to this model, cyclists might not need to pay attention to FoE for guidance compared to drivers due to the slower speed, at which they might have more reaction time to avoid an unexpected hazard and obtain more information with a shorter glance (Vansteenkiste et al., 2013). Another possible reason could be the road condition of an experimental route having one-way straight lanes without curves, in which the road situation can be easily recognized and anticipated with fewer fixations. In these conditions, demand for guidance would diminish.

In contrast to the low demand for guidance, that for stability was greater since cyclists need to maintain their balance themselves by continuing to pedal (Ahlstrom et al., 2016). Additionally, at slower speeds $(<12 \mathrm{~m} / \mathrm{s})$, gazing at nearby regions could play an effective role not only in stability, but in guidance (Land \& Horwood, 1995). Although someone was looking at the road, other information, such as FoE, could be sufficiently understood using the peripheral vision at this speed. Considering the average speed of this study was $3 \mathrm{~m} / \mathrm{s}$, paying attention to the road might be sufficient for obtaining the information necessary for guidance, similar to gazing toward FoE. Therefore, the given conditions would make the gaze strategy using two spots reasonable when cycling.

Third, two specific ranges of eye movement $\left( \pm 30^{\circ}\right.$ horizontally) might be preferred by cyclists in terms of understanding a surrounding environment. While visual information located around a central point $\left(0^{\circ}\right.$ horizontally) was related to navigation and lane-keeping, the ranges of eye movement $\left( \pm 30^{\circ}\right.$ horizontally) had more information on the surrounding situation for detecting hazards or enjoying the streetscape. According to the SEEV model (Horrey et al., 2006), the road user prefers to accept much information with minimal effort, such as slight eye movement, and to gaze at the area where the desired information is expected to be obtained. Considering the efficacy of eye movement, a narrower range than the preferred ranges might not contain the desired information, whereas a wider range might be burdensome in term of cyclists' eye movement. Thus, the two ranges might be suitable for reasonably observing the surrounding situation.

\section{Effects on T-shaped gaze pattern from urban}

characteristics: a raised cycle track, physical separation, land use, and number of pedestrian

In this study, we investigated the effects of urban characteristics on gaze allocation and gaze dispersion. Regardless of the difference of urban environmental characteristics, we could identify the T-shaped gaze area in all situations (see Figure 5). However, different horizontal dispersions were observed according to the presence of a raised cycle track and exclusive bike lanes, even if a distinct dispersion pattern, such as the tunnel vision of drivers (Reimer, 2009; Reimer, Mehler, Wang, \& Coughlin, 2010; Rudin-Brown, Edquist, \& Lenné, 2014), was not found. Our primary findings were as follows.
First, the horizontal gaze dispersion became more active with greater fixations on the all road types including cycle track and sidewalk when participants cycled on non-raised bike lanes. On the other hand, cyclists on a raised cycle track had less active gaze dispersion with more distraction on the building, street furniture and other road users. The main cause of difference of this gaze behavior might be a growing task difficulty. Although both a raised and nonraised cycle track had no difference in the arrangement of road types (including the motorway, bike lane, and sidewalk), the change of road level resulted in different visual street scenes affecting the task difficulty. When cyclists rode a bike on non-raised bike lanes, they might psychologically feel at risk due to the increased mental load from worrying about collisions with cars. Despite the presence of the pavement markings for bicycle paths, this would be insufficient for lessening the burden. Consequently, cyclists would need to spend more time looking at the road with a greater gaze dispersion to avoid a collision.

However, the greater task difficulty always doesn't increase the gaze dispersion. According to the findings of Vansteenkiste et al. (2014), they proposed that gaze dispersion became less active with greater task difficulty. To better explain these different results, what leads to the increased task difficulty of the situation should be considered. In the prior study of Vansteenkiste et al. (2014), the task demand was increased by road quality such as road surface and width, which led to attention being directed to the uneven and narrow road area to check for any obstacles. However, in this study, the psychological burden was caused by the collision risk when participants cycled on non-raised bike lanes, which was more advantageous to widely search for hazards. Thus, we concluded that the gaze dispersion of cyclists is dependent on the road situation and that a high task demand does not necessarily cause a concentrated distribution of fixations.

Second, cycling on the shared bike lanes resulted in a wider gaze movement, but there was not a significantly different fixation percentage on each road factor. Cycling on the exclusive bike lanes doesn't need the active gaze movement than those on the shared bike lanes. This is because an exclusive bike lane with road treatments such as lines, pavement and bollards could provide a more obvious guidance. In addition, cyclists don't need to have greater gaze dispersion on the exclusive bike lanes due to low collision possibility.

However, it was not found the difference of fixation percentage on each road factor even if there was different gaze dispersion. We assumed that it is due to a low number of pedestrians in which participants did not need any clear separation from vehicles and pedestrians. Only the markings of bike paths could be sufficient for safe cycling, not showing the different gaze allocation from cycling in shared bike lanes.

Overall, we found that a gaze pattern could be partially changed within the T-shaped gaze area according to the urban characteristics. It indicates that there is a more suitable gaze behavior, depending on the environmental 
characteristic and it can be beneficial to increase the safety of cyclists. However, less active gaze dispersion and more distraction on the non-related information such as street furniture might lead to another problem. The low gaze dispersion can reduce the detection of a covert and potential hazard. Although cyclists can perceive road situations by using their peripheral vision, focusing on narrow gaze area can be disadvantageous for hazard perception. In addition, distracted gaze behavior also can reduce hazard perception due to cyclists' limited gaze capacity. Therefore, we need to expect the change and limitation of gaze pattern on each road situations and then, additional safety treatments may be necessary in preparation for a risky situation.

\section{Conclusions}

We analyzed gaze movement to explore cyclists' gaze scan patterns and the potential effects of environmental characteristics. Our results demonstrated that cyclists had a Tshaped gaze pattern with two frequent eye fixation points. The pattern may suggest that a T-shaped gaze pattern may benefit cyclists with a greater safety and better readiness to avoid crashes. Furthermore, of the four urban characteristics investigated in the present study, wider gaze dispersion was identified on the shared and non-raised bike lanes. We identified that there is a more suitable gaze behavior, depending on the environmental characteristic and it can be beneficial to increase the safety of cyclists.

The eye-tracking methodology used in this paper was effective to investigate cyclists' eye movement. Unlike previous measurements such as survey and video analysis, the eye tracker could exactly detect where cyclist focus on. However, a gaze point measured by an eye tracker may not indicate that it is perceived by cyclists. This is because people sometimes unconsciously look at something when cycling. Then, it may not be clear to conclude that we can find the attention point by using an eye-tracker. Despite this limitations, the gaze position is generally interpreted to have a high possibility to be perceived. We could assess which road factors have a potentially important role by using this method. In addition, the limitation could be reduced by carefully adjusting an eye movement data.

The findings in cyclists' gaze pattern lead to the following urban planning and design implications. First, bicycle facilities have to be planned according to the consideration of the T-shaped gaze area. We identified that there were specific areas and spots having the greater possibility to be gazed. Considering these areas and spots can enhance the effect of present bicycle facilities. For example, bicycle signs are important to notify information about a road situation. Different types of sign (such as bike road markings, post-mounted signs, and overhead signs) have been constructed in many countries. However, not all signs are recognized to the same degree when cycling. The gaze possibility is different, depending on the location of signs. Signs that are placed within cyclists' frequent gaze area can be easily detected by cyclists. This means that a road marking can be more useful than an overhead sign for detection by cyclists since a road marking is within the T-shaped area, whereas an overhead sign is not. Thus, placing bicycle treatments at a suitable location may improve information delivery more effectively by expecting where cyclists will frequently look.

Second, a specific gaze behavior at a particular road environment may need additional safety treatments. We found that cyclists on the raised and exclusive cycle track had less active gaze movement. In addition, cyclists on the raised cycle track had greater distraction to the external area such as building and vehicles \& pedestrians. In this case, these gaze pattern sometimes may reduce the detection of a sudden risky situation since people have a limited capacity to look around (Mantuano et al., 2017). Cyclists with less active gaze dispersion may have a greater risk involving an accident than those with wider gaze dispersion. It means that additional treatments may be needed to increase cyclists' hazard perception on the road that leads the less active gaze dispersion. For instance, more signs that can notify unexpected hazards may be beneficial on the raised cycle track by reducing the limitations of narrow gaze dispersion. Moreover, repeated arrangement of a shared and an exclusive bikeway may be effective by adjusting cyclists' gaze dispersion. When hazards are expected and a wider gaze dispersion is needed, changing a part of exclusive bike lanes to shared bikeways can lead cyclists to actively look around. Thus, urban planners need to consider the different gaze pattern and limitation on each road environment when they construct bicycle facilities. In addition, road environment has to be planned to lead cyclists to take a safety gaze behavior.

The present study's value lies in that it contributes towards a better understanding of cyclists' gaze pattern in a real environment, which provides meaningful implications for improving cycling infrastructure. However, one methodological limitation of the present study is that the analysis generally included no consideration of unexpected road situations. Since the proposed gaze pattern results were obtained in controlled urban situations, another limitation is that our results may still not insufficient to effectively predict various changes in diverse urban situations. Furthermore, the quite high standard deviations in our results suggest that other factors (such as age, skill, and speed) should also be considered alongside environmental factors.

Therefore, we propose that a future research needs to consider these limitations. The gaze pattern in an intersection has not been discovered even though a lot of accidents in the intersection have happened. Since cyclists' gaze behavior in the intersection is expected to have an obvious difference with those on a straight road, we need to investigate the cyclists' gaze pattern in the intersection in the future. Moreover, combining an eye tracker with other methodologies such as EGG, emotion recognition and interview can enhance the accuracy of the measurement of gaze behavior. We could track and record eye positions with an eye-tracker but we didn't know exactly why cyclists looked at a particular point. Then, an additional measurement can reduce this limitation by recording 
other information such as participant's emotion and memory. Thus, considering a variety of methodologies can be helpful to better investigate cyclists' gaze behavior.

\section{Author contributions}

Gukhwa Jang contributed to the whole work including design of the data analysis, data collection, data analysis, interpretation and writing the article. Saehoon Kim was also responsible for data interpretation and development of manuscript of the article.

\section{References}

Ahlstrom, C., Kircher, K., Thorslund, B., \& Adell, E. (2016). Bicyclists' visual strategies when conducting self-paced vs. system-paced smartphone tasks in traffic. Transportation research part F: traffic psychology and behaviour, 41, 204-216. https://doi.org/10.1016/j.trf.2015.01.010

Ballham, A., Absoud, E. M., Kotecha, M. B., \& Bodiwala, G. G. (1985). A study of bicycle accidents. Injury, 16(6), 405-408. https://doi.org/10.1016/0020-1383(85)90057-9

Bendak, S., \& Al-Saleh, K. (2010). The role of roadside advertising signs in distracting drivers. International Journal of Industrial Ergonomics, 40(3), 233-236.

https://doi.org/10.1016/j.ergon.2009.12.001

Chapman, P., Underwood, G., \& Roberts, K. (2002). Visual search patterns in trained and untrained novice drivers. Transportation Research Part F: Traffic Psychology and Behaviour, 5(2), 157-167. https://doi.org/10.1016/S1369-8478(02)00014-1

Crundall, D., Van Loon, E., \& Underwood, G. (2006). Attraction and distraction of attention with roadside advertisements. Accident Analysis \& Prevention, 38(4), 671-677. https://doi.org/10.1016/j.aap.2005.12.012

Donges, E. (1978). A two-level model of driver steering behavior. Human factors, 20(6), 691-707. https://doi.org/10.1177/001872087802000607

Edquist, J., Horberry, T., Hosking, S., \& Johnston, I. (2011). Effects of advertising billboards during simulated driving. Applied ergonomics, 42(4), 619-626.

https://doi.org/10.1016/j.apergo.2010.08.013

Frings, D., Parkin, J., \& Ridley, A. M. (2014). The effects of cycle lanes, vehicle to kerb distance and vehicle type on cyclists' attention allocation during junction negotiation. Accident Analysis \& Prevention, 72, 411-421. https://doi.org/10.1016/j.aap.2014.07.034

Helander, M., \& Söderberg, S. (1972). Driver visual behavior and electrodermal response during highway driving. University of Göteborg, Department of Psychology.

Hughes, P. K., \& Cole, B. L. (1986). What attracts attention when driving? Ergonomics, 29(3), 377-391. https://doi.org/10.1080/00140138608968272

Hajime, I. T. O., Atsumi, B., Hiroshi, U. N. O., \& Akamatsu, M. (2001). Visual distraction while driving: trends in research and standardization. IATSS research, 25(2), 20-28. https://doi.org/10.1016/S0386-1112(14)60067-4

Horrey, W. J., Wickens, C. D., \& Consalus, K. P. (2006). Modeling drivers' visual attention allocation while interacting with in-vehicle technologies. Journal of Experimental Psychology: Applied, 12(2), 67. https://doi.org/10.1037/1076-898X.12.2.67

Hosking, S. G., Liu, C. C., \& Bayly, M. (2010). The visual search patterns and hazard responses of experienced and inexperienced motorcycle riders. Accident Analysis \& Prevention, 42(1), 196-202. https://doi.org/10.1016/j.aap.2009.07.023
Land, M., \& Horwood, J. (1995). Which parts of the road guide steering? Nature, 377(6547), 339-340. https://doi.org/10.1038/377339a0

Liu, X., Shen, L., \& Huang, J. (1995). Analysis of bicycle accidents and recommended countermeasures in Beijing, China. Transportation research record, 1487, 75-83.

Mourant, R. R., \& Rockwell, T. H. (1970). Mapping eye-movement patterns to the visual scene in driving: An exploratory study. Human factors, 12(1), 81-87. https://doi.org/10.1177/001872087001200112

Mantuano, A., Bernardi, S., \& Rupi, F. (2017). Cyclist gaze behavior in urban space: An eye-tracking experiment on the bicycle network of Bologna. Case studies on transport policy, 5(2), 408-416. https://doi.org/10.1016/j.cstp.2016.06.001

National Association of City Transportation Officials. (2014). Urban bikeway design guide. Island Press.

Pelz, J. B., \& Rothkopf, C. (2007). Oculomotor behavior in natural and man-made environments. In Eye Movements (pp. 661676). Elsevier.

Perez, W., \& Bertola, M. A. (2011). The effect of visual clutter on driver eye glance behavior. In Proceedings of the Sixth International Driving Symposium on Human Factors in Driver Assessment, Training and Vehicle Design (pp. 180-186). Lake Tahoe, California. Iowa City, IA: Public Policy Center, University of Iowa. https://doi.org/10.17077/drivingassessment.1395

Reimer, B. (2009). Impact of cognitive task complexity on drivers' visual tunneling. Transportation Research Record: Journal of the Transportation Research Board, 2138, 13-19. https://doi.org/10.3141/2138-03

Reimer, B., Mehler, B., Wang, Y., \& Coughlin, J. F. (2010). The impact of systematic variation of cognitive demand on drivers' visual attention across multiple age groups. In Proceedings of the Human Factors and Ergonomics Society Annual Meeting, 54(24), 2052-2055. Sage CA: Los Angeles, CA: SAGE Publications. https://doi.org/10.1177/154193121005402407

Rudin-Brown, C. M., Edquist, J., \& Lenné, M. G. (2014). Effects of driving experience and sensation-seeking on drivers' adaptation to road environment complexity. Safety Science, 62, 121-129. https://doi.org/10.1016/j.ssci.2013.08.012

Vansteenkiste, P., Cardon, G., D’Hondt, E., Philippaerts, R., \& Lenoir, M. (2013). The visual control of bicycle steering: The effects of speed and path width. Accident Analysis \& Prevention, 51, 222-227. https://doi.org/10.1016/j.aap.2012.11.025

Vansteenkiste, P., Zeuwts, L., Cardon, G., Philippaerts, R., \& Lenoir, M. (2014). The implications of low quality bicycle paths on gaze behavior of cyclists: A field test. Transportation research part F: traffic psychology and behaviour, 23, 81-87. https://doi.org/10.1016/j.trf.2013.12.019

Vansteenkiste, P., Cardon, G., \& Lenoir, M. (2015). Visual guidance during bicycle steering through narrow lanes: A study in children. Accident Analysis \& Prevention, 78, 8-13. https://doi.org/10.1016/j.aap.2015.02.010

Vansteenkiste, P., Zeuwts, L., Cardon, G., \& Lenoir, M. (2016). A hazard-perception test for cycling children: an exploratory study. Transportation research part F: traffic psychology and behaviour, 41, 182-194. https://doi.org/10.1016/j.trf.2016.05.001

Vanparijs, J., Panis, L. I., Meeusen, R., \& de Geus, B. (2016). Characteristics of bicycle crashes in an adolescent population in Flanders (Belgium). Accident Analysis \& Prevention, 97, 103-110. https://doi.org/10.1016/j.aap.2016.08.018

Zeuwts, L. H., Vansteenkiste, P., Deconinck, F. J., Cardon, G., \& Lenoir, M. (2017). Hazard perception in young cyclists and adult cyclists. Accident Analysis \& Prevention, 105, 64-71. https://doi.org/10.1016/j.aap.2016.04.034 